\title{
Josef Brink Der Funktionswandel des Strafrechts in der Stalin-Zeit
}

Die Stalin-Zeit isc ohne ihre strafrechtliche Dimension, insbesondere ohne den Funktionswandel des sowjetischen Strafrechts nicht zu begreifen. Um nicht der Versuchung zu erliegen, jenseits der Empirie einen solchen Funktionswandel aus dem »Wesen* des Stalinismus und der diesen bestimmenden spezifischen sozialökonomischen Beziehung der Staatsmacht zur gesellschaftichen Produktion a priori herzuleiten, wählt die Untersuchung den Moskauer Prozeß von $1938 \mathrm{zu}$ ihrem phänomenologischen Ausgangspunkt. Diese Wahl erfolgt nicht aus der Verlegenheit, willkürich einen Ausschnir der Rechtsanwendung in der Stalinzeit als repräsentariv auszugeben. Der Prozeß von 1938 gegen Bucharin, Rykow, Rakowsky und zwanzig weitere ehemals fühurende Bolschewiki ist der Kulminarionspunkt der politischen Justiz der dreißiger Jahre in der Sowjerunion. Parallel zum Höhepunkt von administrativen Säuberungen in Partei, Verwaltung, Industrie und Armee findet hier das stalinistische Strafrecht in Aktion sein paradigmatisches Niveau. In dem Prozeß sind die Anklageformeln aller Schädlings-, Diversions-, Spionage- und Hochverratsprozesse seic 1928 kumulativ aufgehoben.'

Ausgegangen wird von der spezifischen strafrechtichen Form des Prozesses gegen Bucharin und andere führende Bolschewiki. Der Versuch, den Moskauer Prozeß von seinen normativen, positiv-rechtlichen und rechtsdogmatischen Determinanten her anzugehen und den Strafrechrstypus der Stalinzeic in seiner Anwendung wie in seinen Resultaten als juristische "Bewältigung" gesellschaftlicher Widersprüche zu begreifen, ist bisher weder von der juristischen Literarur, die sich mit dem sowjetischen Rechtssystem beschäfrige, noch von der politikwissenschaftlich orientierten Literatur, die sich dem Phänomen der politischen Jusciz dieser Zeic zugewandt hat, unternommen worden.

Von juristischer Seite hat man sich bisher im wesentlichen darauf beschränkr, den Gang der sowjetischen Rechtsentwicklung positivistisch nachzuzeichnen und unter verschiedenen Fragestellungen zu systematisieren. Die politikwissenschafrliche und historische Literatur hat sich bis auf wenige Ausnahmen darauf beschränkt, das Material in Form einer chronologisch organisierten Ereignisgeschichte zu präsentieren', in allgemein gehaitenen Beschreibungen Mechanismen der sowjerischen politi-

1 Vgl.W. Ziehr, Dic Entwicklung des ,Schauprozesses, in der Sowjetunion. (Ein Bcitrag zur sowjecischen Innenpolizik 1928-1938), Diss., Tübingen 1969.

a Vgl. R. Maurach, Das Rechrssystem der UdSSR, München 1953: Geilke, Einführung io das Sowjeurecht, Darmstadt 1966; W. Mcder, Das Sowjetrecht, FrankfurdM. Berlin 1971; B. Meissner, Entpricklung und Gründzüge der sowjeoschen Staseslehre, und F. C. Schroeder, Fünizig Jahre sowjccische Rechtsucorie, in: R. Maurach / B. Meissner ( $\mathrm{Hg}$.), so Jahre Sowjeưecht, Stuugarh Berlin, Köln, Mainz ig6g.

3 Diese hisorische Zugangsweise öählen T. Pirker, Die Moskauer Schauprozesse 1936-1938, München 1963: G. Dickler, Oie Moskauer Prozesse (1936-1938) in: ders., Prozesse, dic Geschichuc machuen. FrankfurdM.- Hamburg 1966, S. 9s If.; R. Conquese, Am Anlang starb Genosse Kirow. Säuberungen unter Stalin, Düsscldont 1970. 
schen Justiz herauszuarbeiten^ oder einige, besonders hervorstechende prozessuale Takciken zu bezeichnen'. Bei der Frage nach den politischen und sozialen Gründen für die Durchführung des Prozesses im Jahre 1938 verliert sich diese Literatur in der Regel in personalisierenden, psychologisierenden und moralisierenden Betrachtungen $^{6}$, die allesame schließlich nur Unerklärliches in der Tatsache finden können, daß ehemals führende Bolschewiki als gemeine Verbrecher sich verantworten müssen. Dieses gilr in gleicher Weise für westliche Autoren, die sich im Bezugsrahmen der sogenannten Toralitarismus-Theorie bewegen, wie für die östlichen Autoren, die unter dem restringierten Bezugsrahmen eines Krivik des Personenkules an das Material herangehen.?

Aus der strafrechalichen Form des Prozesses und der Analyse des Funktionswandels des angewandten Strafrechts erschließen sich jedoch ersz die sozialen und politischen Gründe für die Inszenierung des Moskauer Verfahrens. Die Erkennenis der neuen Sozialgestatrungsfunkrion des sowjerischen Surafrechts erlaubr es, die sachverhaitlichen Anknüpfungspunkce und das Resulcat des Prozesses nicht einfach als absurden Racheplan ${ }^{8}$ Stalins anzusehen, sondern sie als Verarbeitung real-historischer Probleme der dreißiger Jahre im scrafrechtlichen Gewande ernst zu nehmen.

\section{Die strafrechtliche Form des Moskaner Prozesses}

In der Literanur zum Phänomen der Moskauer Prozesse ist immer wieder der Versuch unternommen worden, die sachverhaleliche Grundlage und Stimmigkeir der Anklage keritisch zu überprüfen und die Anklage als blanke "Justizfälschung" " kenntlich zu machen. Ein Nachweis, daß die Anklagen gegen prominente Bolschewiki so absurd seien, sdaß nur ein monomanisches Gehirn sie erfunden und ein korrumpiertes sie für wahr halten konntea ${ }^{10}$, wie Theo Pirker resümiert, rrifft aber noch nicht die eigencliche Ebene des Problems. Es liegr zwar auf der Hand, daß es sich bei dem Moskauer Prozeß von 1938 um einen politischen Prozeß handelt, bei dem die Polirik den Ton angibr, das Strafrecht allenfalls als Mitrel der Sprachregelung und Rechtfertigung erscheint. Diese politische Dimension wird im Prozeß selber jedoch kaum deutlich. Dieser gerierr sich apolitisch und gänzlich juriscisch.

\ So an Besspielen O. Kirchheimer, Politisehe Jusiz, Verwendung juristischer Verfahrensmöglichkeimn zu policischen Zwecken, Neuwied-Berlin 1969, S. 166 If.

s So: N. Lcires / L. Bcrnaud, Rinual of Liquidaijons. The Case of the Moskow Trials, Glencoe 1954, 5. $350 \mathrm{ff}$.

6 Personalisierende, psychologisierende und moralisierende Erklarungs- und Darsiellungsweisen linden sich bei: Paschner, Im Tculelstereis des Terrors, Boppard 1964, S. 73 ff.; G. Dickler, 2. a. O., S. 100 1.; $H$. Arend, Uber dic Revolution, München 1974, S. 70 Il.; J. Carmichael, Säuberung. Die Konsolidie. rung des Sowjetregimes unter Stalin 1934/38, Frankfurd/M.-Berlin-Wien 1972, S. 2 il ff.

7 In einzigareiger Weisc hal demgegcnüber M. Merleau-Pontys Schrif -Humasisnus und Tenor" (FrankfurdM. 1966) die politisethe Jusciz der Sialin-Zcit zum Gegenstand der Analyse gemache, indem sie das singulärc Ereignis der strafrechelichen Poenalisierung aus seiner Isolięrung laste und zu der Gesellschaft in Bezichung setzte, dic sich solcher Justiz bedienre. Nicht im Engebnis, aber im Zugang und der Problemsceilung knüph der Autor an Merleau-Pontys Reflexionen an. Im Resultat weiß er sich R. Bahros Altemacive. Zur Kririk des real-exisierenden Sozialismus* (Frank/ur/M.-Köln 1977) verpllichtet.

8 So sogar die große Arbeit R. Medwedjcws "Die Wahrheil ist unscre Stärke = (Frankfurt/M. 1973, S. 2 14): - Stalins eigendiches Motiv hieß Rache, Rache an seinen ehemaligen politischen Gegnern, die gelegentlich lose Bernerkungen gemachr harten. In den zocr Jahren hatre es Scalin an der Macht gefehlt, sich physisch an ihnen zu rächen. Er warece scine Zcit ab. (...) Und als er sich stark genug dazu fühlee, vernichtere er die Aksivisten der eliemaligen Opposicionsgruppen. a Medwedjew verbleibr damir im Bezugsrahmen der haibherzigen Geheimrede Chruschichows vom 25. Februar 1956, in: Medwedjew U. 2., Enstalinisierung. Der XX. Pancitag der KPdSU und seine Folgen, Frankfurd'M. 1977, S. 491. 9 L. Troezki, Sealins Verbrechen, Berlin 1973, S. 229.

10 T. Pirker, Die Moskauer Sehauprozesse 1936-1938, Munchen 1963, 5. 72. 
Die strafrechtliche und strafprozessuale Dimension ist die eizzige Darstellungsform des Prozesses. Sie ermöglichr die Durchsetzung der politischen Zrvecke. Als Produkrionsprozeß von Interpretationen und Argumentacionen muß die Rechtsförmigkeit des Moskauer Prozesses daher ernst genommen werden. Als Darstellungsmittel offenbart sie die Zwecke des Verfahrens.

\section{Zur Entstehung des stalinistischen Sirafrechis}

Der Typus des Strafrechts, das den Moskauer Prozeß von 1938 materiell-rechulich dererminiert, weicht in seinen elementaren Bestimmungen von den Prinzipien des revolutionären Strafrechts der ersten Jahre nach der Oktoberrevolution ab. Dieses zielte als Maßregelstrafrecht mit den Strafzwecken der Besserung und Erziehung jedenfalls seinem Anspruch nach und - soweit es mit gemeiner Kriminalität von Tätern aus dem Proletariac konfroncier war- z. T. auch in der Praxis darauf ab, sich gleichsam mit der Entwicklung der entfaltecen sozialistischen Gesellschaft überflüssig zu machen.

Als es mit den, Leitenden Grundsätzen für das Surafrecht der RSFSR، vom 12. 12. 1919 zu einer ersten Kodifizierung von Strafzweckbestimmungen kam, wurden als Strafzwecke nicht Sühne, Vergeltung oder generelle Abschreckung, sondern MaBnahmen der Besserung und Anpassung zur Sicherung der Macht des Proletariats festgesetzt. Auch das Strafgeseczbuch der RSFSR vom 1. 6. 1922, das die ,Grundsäzze ablöste, wie das Strafgeserzbuch der RSFSR vom 22. 11. 1926 gingen konsequent bei der Bestimmung der Kodifikationszwecke vom Grundsatz der spezialpräventiven Gefahrenabwehr aus. Das SiGB von 1926 sah sich als "Strafreche ohne Schuld und Strafe ${ }^{1}$, das anstelle des bürgerlichen Vergetungsstrafrechts Maßregeln der Besserung, Sicherung und des sozialen Schutzes stelle, die myrhologische Kategorie der Schuld als Strafzumessungskriterium durch die Kategorie der ssozialen Gefährlichkeit ersetzte und das Maurach deshalb als sdie leczte Radikalisierung des Strafrechos "' bezeichner. Wenn dieses Strafrecht auch den Horizont des bürgerlichen Rechrs nicht sprengte, so zielte es wie die Rechestheorie der zoer Jahre von Stučka und Paschukanis doch in diese Richtung. Das sich ab 1927/28 mit der staaclichen Unionsgesetzgebung durchsetzende Strafrecht kehrt dagegen zu einem Bestrafungsrecht mit dem Strafzweck eindeutiger Generalprävention zurück." Es nimms den bürgerlichen Strafzumessungsgesichtspunkt der

Ir R. Maurach. Das Rechessysicm der UdSSR, München 1993, S. 13.

12 Ebenda, S. 19; ebenso: G. Geilke, Einführung in das Sowjetrecht, Darmsuade 1966, 5. ,6 6f.; W. Mcder, Das Sowjeureche, Frankfur/M.-Berlin 1971, S. $190 \mathrm{H}$.

1) Vor allem dic Unionsgeselzgebung, die sich mit der Ausweinng der Herrschafi Scalins forcien entwickeltc, obwohl die Union zunächst auf die Grundlagengesezzgebung beschränkt war, hatte eine cigenständige, von der des wcitcrhin geltenden Republikenstrafrechts abweichende Tendeaz. So sah z. B. die Verordnung des ZEK und des Raes der Volkskommissare vam 7. August 1932, Uber den Schurz des Vermögens von staatichen Unternehmen, Kolchosen und Genossenschaften und die Ferigung des gesellschafdichen sozialistischen Eigenturns, bei Traosportberaubung und Diebstakl von suatlichem und genossenschafitichern Vermögen die höchste Surafe, den Tod, und die Vermögenskon. fiskzion vor. Dic Verordnung des ZK der KPdSU und des Rats der Valkskommissare der UdSSR vom 31. Mai r939 bescimmec, daß zur Bekämplung von Jugendbanden Minderjährige nach Vollendung des 12. Lebensjahres für bestimmic Serafiaten nach Eroacbsenenrecht besiraft overden mußien. Das politische Stralrecht, wie es auch im Moskauer Prozeß zur Anwendung gelangte, wurde in scinen entscheidenden Teilen verschärh: Durch die Verordnung des ZEK der UdSSR vom 8. Juni 1934 wurde Landesverrach Spionage und Fluche ins Ausland mie der Todessisáfe belegr, den Angehörigen von Auslandstlüchógen, dic dic Fluchabsichr gefördcrt oder, obwohl sie davon Kennmis haren, niche angezcigr hanen, eine zehnjährige Freiheitsstrafe und Vermögenscinziehung angezeigz. Den Umbruch in der Rechtsennvicklung der Sowjetunion in den Jahren 1927/28 siehs auch D. Pfaff, Die Enrwicklung der sowjerischen Rechuslehre, Xöln 1968, S. 87. 
Schuld wieder auf, erhöht die angedrohten Srrafisanktionen bis ins Ungeheuerliche und weiret seine soziale Zugriffskapazitär radikal aus.

Das wird vor allem im Kernbereich des gesellschaftlichen Lebens, an der juristischen Stellung der Produzenten im Produkcionsprozeß deutlich. Die Verschärfung arbeitsrechtlicher Disziplinarmaßßnahmen, das Verbor des Arbeitsplatzwechsels, die Inpflichtnahme jedes Arbeiters für den reibungslosen Ablauf der Produktion durch die Arbeitsgeserzgebung ab dem Jahre 1929 und das strikte Arbeitsgebor der Verfassung von 1936 stiften eine strafrechtlich bedeutsame Verpflichtungs- und Garantenstellung eines jeden Arbeiters, die gleichsam jede Abweichung des Produktionsprozesses von Leistungs- oder Standardnormen, selbst wenn sie Folge einer übereilten und daher gewaltsamen Industrialisierungspolicik ist, zu einer individuell zu verantwortenden Devianz, im strafrechtlichen Sinne zu einem sogenannten unechren. Unterlassungsdelikt stilisierr. Die Ausstrahlung der Arbeitspilicht führn zu einem Bedeutungswandel der im Strafgesetz kodifizieren Kommissivdelikre zu gleichsam heimlichen Ommissivdelikten, der eine erhebliche Vergrößerung der Eingriffsfelder der Strafjusciz bewirkt.

Diese dem Strafrecht unterlegte Dominanz von Gebotsgehalten gegenüber solchen des Verbots wird in der stalinistischen Rechtstheorie zum Strukturprinzip erklärt. Von A. J. Wyschinskij wird Pflichcerfüllung, nicht eben nur bloße Abstinenz von gesellschaftsschädlichem Verhalten, als Normzweck des Strafrechts gesehen. «Das sowjerische Strafrecht erhebr im Namen des Sowjetstaaces die Fordenung nach bestimmrem Verhalten, nach einer bescimmeen Einstellung zu den Pflichten der Sowjerbürger, indem es die Erfüllung dieser Pflichten unter Strafandrohung für verbindlich erklärt. $x^{14}$ Das Strafrecht critt damit aus seiner peripheren Funktion der bessernden Behandlung des einzelnen devianten Subjekts, die auf die Aufhebung der Rechtsverhältnisse bei der Regulierung der gesellschaftlichen Verhältnisse zielte, heraus und gewinn die Rolle eines Gestaltungsmittels für dîese Verhältnisse. Es wird, wie Wyschinskij es formulierte, zu cinem ${ }^{2}$ Hebel der Verwaltung «.'

Dieselbe Entwicklungstendenz finder sich auch in der Unionsgeserzgebung zum Strafverfahrensrecht. Am weitesren reicht auf diesem Rechesgebiet eine Beschleunigungsnovelle anläßlich der Ermordung des Politbüromitglieds Kirow. Der Mörder Kirows, eines engen Vertrauten Sialins, war der junge Kommunist Nikolajew, der auch bereits in einem Schnellverfahren im Dezember 1934 verurteilt und hingerichtet wurde. Das noch am Tage von Kirows Tod sogleich verkündete Sondergesetz ,Úber die vereinfachte Strafverfolgung terroristischer Organisationen und terroristischer Verbrechen vom I. Dezember 1934 sah bei Verfahren wegen terroriscischer Akte gegen Sowjetfunkcionäre die Verfahrensdurchführung binnen zehn Tagen vor, schloB Rechtsmittel und Gnadengesuche gegen das Urreil aus und ordnete die sofortige Vollstreckung der Todesstrafe nach der Urteilsverkündung an. ${ }^{16}$

\section{Das stalinistische Strafrecht und die Anklage im Moskauer Prozeß}

Die grundlegende Norm für den Moskauer Prozeß von 1938 war der Arrikel 58 des Stralgeserzbuches mit seinen Erweiterungen durch die Verordnung des ZEX vom 8. Juni 1934. ${ }^{17}$ Artikel 58 , der als Straftarbestand zu einem Teil mir den Unionsge-

14 A. J. Wyschinskij. Fragen des Rechis und des Staztes bei Marx, in: Sowjetische Beiträgc zur Staats- und Rechisuheoric, Berlin 19;3, S. 39.

is Ebenda, S. Is.

16 Vgl. W. Meder, 2. 2. O., S. 210; G. Geilke, 1. 2. O., S. 89; R. Maurach, a. 2. O., S. 42.

17 Zur Anwendung gelangen dic Aruikel $58-1 a, 5^{8-7},\left\{^{8-8}, 5^{8-9}, 5^{8-1}\right.$ ) und $\left.5^{8-1}\right\}$ des StGB der RSFSR. 
setzen über die Staatsverbrechen vom 28. Februar 1927 und über die Militärverbrechen vom 27. Juil 1927, zum anderen Teil durch die genannte Verordnung vom 8. Juni 1934 in das StGB der RSFSR vom 22. November 1926 erst aúgenommen worden war, erfaßte die sogenannten agegenrevolucionären Verbrechen *. ${ }^{8} \mathrm{Als}$ solche definierte er alle Handlungen, wdie auf den Sturz, die Unterhöhlung oder die Schwächung ${ }^{19}$ der inneren Verfassung und äußeren Sicherheit der Sowjetunion gerichtet waren und spezifizierte diesen Grundtatbestand dann im einzelnen als Varerlandsvertar (Spionage, Geheimnisverrac), ais bewaffneten Aufstand, als Unterhöhlung der industriellen Entwicklung, als "Begehung terroriscischer Handlungen $\alpha^{20}$, als win gegenrevolutionärer Absicht mirtels Sprengung, Brandstiftung oder auf andere Weise durchgeführe Zerstörung oder Beschädigung " ${ }^{21}$ von öffenclichen Anlagen, dann als njede organisatorische Tärigkeit, die auf die Vorbereitung oder Begehung eines gegenrevolucionären Verbrechens gerichret isc " ${ }^{21}$ und schließlich in Art. 58-13 als saktive Handlungen oder aktiver Kampt gegen die Interessen der Arbeiterklasse und der revolucionären Bewegung a's. Als Strafmaß schrieb der Art. s8-ra die "Erschießung verbunden mit der Konfiskation des gesamten Vermögens oder beim Vorliegen mildender Umscände . . 10 Jahre Freiheicsentzug verbunden mit Konfiskarion des gesamten Vermögens $\alpha^{24}$ vor.

Die auf dieser Grundlage erstellte Anklageformel im Moskauer Prozeß warf den Angeklagren die Bildung einer Verschwörergruppe unter dem Namen, Block der Rechten und Trotzkisten« vor, "die sich zum Ziel setzte Spionage zugunsten auswärtiger Sraaten, Schädlingstätigkeit, Diversion, Terrorakte, Untergrabung der Wehrkraft der UdSSR, Provozierung eines milicärischen Uberfalls dieser Scaacen auf die UdSSR, Niederlage der UdSSR, Zerstückelung der UdSSR und Lostrennung der Ukraine, Belorußlands, der Mirtelasiatischen Republiken, Georgiens, Armeniens, Aserbaidshans, des Fernöstlichen Küstengebiets (Primaje) von ihr zugunsten der erwähnten auswärrigen Staaten und endlich der Sturz der in der UdSSR bestehenden sozialistischen Gesellschafis- und Staatsordnung und die Wiederherstellung des Kapicalismus, die Wiederherstellung der Machr der Bourgeoisie. $\alpha^{25}$

Die Angeklagten seien mit ausländischen Staaten in Verbindung getreten, um von ihnen militärische Hilfe zu erhalten, hätten systemacisch in den "Zweigen des sozialistischen Aufbaus ${ }^{26}$ der Industrie, der Landwirtschafr, im Eisenbahnverkehr, der Finanz- und Kommunalwirtschaft Schädlings- und Diversionsakte ausgeführt. "Ohne jede Stütze innerhalb der UdSSR $\alpha^{27}$ hätten die Angeklagten zunächst eine Spionagetärigkeic für ausländische Staaten aufgenommen.

Dann seien sie dazu übergegangen, mit Deutschland, Japan, Polen und England Absprachen bezüglich der Abrrennung von sowjetischen Gebieten nach einem Krieg mit der Sowjetunion zu treffen. Japan sei Usbekisian, Deutschland die Ukraine und Polen Belorußland versprochen worden. Zugleich hätten die Angeklagten Diversions- und Schädlingsakte gegen die sowjerische Wirschaft unternom-

Siehe: Prozeßbericht über die Strásache des ancisowjecischen 'Blocks der Rechten und Troızkistene, Volkskommissariat fur Justizwesen der UdSSR ( $\mathrm{Hg}_{\mathrm{g}}$ ), Vollstündiger sienographischer Berich, Moskau 1938, S. 37 .

18 Siehe W. Meder, 2. 2. O., 5. 19s zu Art. $5^{8-1}$ StGB

ig Zir, nach W. Meder, a. a. O., S. 19s.

20 Nach ebenda, S. 198, Arr. s8-8.

21 Nach ebenda, S. 19\$, Ar. $58-9$.

22 Nach ebenda, S. 199, Arr. 58-11.

23 Nach ebenda, S. 199, Art. 58-13.

${ }_{4}$ Nach ebenda, 5. 196, Art, 58-ra.

2s Prozeßbericbr, a. 2. O., S. 36.

26 Ebenda.

27 Ebenda, S. 6. 
men, um die Verteidigung der Sowjetunion zu schwächen. "Sie machten es sich zur Aufgabe, das ganze wirtschaftliche Leben des Landes sowie die Versorgung der Armee mit Lebensmitreln und Waffen lahmzulegen. ${ }^{28}$

Schließlich, als diese Maßnahmen nicht zum gewünschten Erfolg geführt hätten, seien die von Wut und $\mathrm{Ha}$ gegen die UdSSR erfültten rechten und trotzkistischen Verschwörer dazu übergegangen, Terrorakte gegen die Regierung und die KPdSU(B) vorzubereiten und durchzuführen. «" ${ }^{29}$ Folgerichrig hä̈ten die Angeklagten in den zoer Jahren dann die Ermordung Stalins, Molotows und der gesamten Partei- und Staatsführung vorbereitet und das Politbüromitglied Kirow dadurch, daß der Angeklagte Jagoda als zuständiger Kommissar dem Mörder Nikolajew keine Hindernisse in den Weg legte, auch ermorder. Diese Art von Arklage, die mit dem Vorwurf gegen den früheren Parteisekretär Selenski, vom Jahre ig1x an Agent der zaristischen Geheimpolizei Ochrana gewesen zu sein, oder gegen Bucharin, im Jahre 1918 Mordpläne gegen Lenin gefaßs zu haben, die verbrecherische Natur der Angeklagten gleichsam schon in der Wiege suchre, isc nicht mehr von einer offenen, auf die Zukunfe gerichteten Argumentation beherrscht, wie sie bis zu einem gewissen Grade noch in den politischen Verfahren der frühen und micteren zwanziger Jahre zu beobachten war..$^{10}$ In den Prozessen dieser Zeit arurde offene Innenpolitik oder offene Außenpolitzk gemacht, die mit auf die politische Zukunft gerichıeren Gefährlichkeitsprognosen argumentierte. Selbst wenn man diese Politik ablehne, so muß man doch konzedieren, daß sie sich ais von einer sozialiscischen, erst zu verwirklichenden Zukunftsperspektive abgeleitere und legitimierte, explizit politische Argumentarion deuclich machee.

Die AnkJage im Moskauer Prozeß stellt sich dagegen nicht mehr als eine Form politischer Auseinandersetzung dar, sondern beschwörn selekriv die verbrecherische Vergangenheit der Angeklagten im gemeinen Sinne. Das Strafrecht wird zum Lebensführungs- und Charakterstrafrecht, wie, verborgen unter dem mythologischen Begriff der Schuld, das bürgerliche Sirafrecht. Die Anklage vermeidet jeden Bezug auf politische, von den Angeklagren repräsencierte Konzeprionen. Allein die private Verbrechernatur der Angeklagien ist ihr Gegenscand. Für den Ankläger Wyschinskij vertraren diese denn auch auch gar nichts »Ideelles, nichts sozusagen - Geistigesu, niches Ideologisches. Was einst in irgendeinem Maße einige von den Teilnełmern dieses Blockes besaßen, das ist schon längss verloren, eingebüßt, längst verschwunden, verwest in dem verpesteren, widerlichen unterirdischen Treiben der Spione. « ${ }^{\prime \prime}$

\section{Die Diffusion des juristischen Teilnabmebegriffs}

Gemessen an den Tarzusammenhängen, die den Angeklagren von der Anklage als Verbrechen zugerechnet wurden, stellen diese sich als eine äußerst heterogene Gruppe dar. Was auf der Ebene von Tatsachen das Verbindende und Gemeinsame dieser Gruppen ausmacht, kommt in der Anklage nicht zum Ausdruck. Allein auf der Ebene von ,Oriencienungen erscheint gleichsam der Nutzen des Auslands von all den Akrivitäten der verschiedenen Angeklagtengruppen als verbindendes Element, das alle Angekdagten scheinbar zu einer zielgerichteten Organisation zusam-

28 Ebenda, S. Is.

29 Ebcinda, S. 21 I.

jo Siehe W. Zichr, a. 2 O., S. 18 ff.; auch zum Prozeß gegen dic Sozialrevolucionäre Parei, S. 45 f,, und zum Prozeß gegen Kindermann, S. $88 \mathrm{f}$.

31 Prozeßbericht, a. a. O., S. 678. 
menfaßs. Enssprechend diffus und tentativ fallen dann auch die Begriffe aus, mir denen der Staatsanwalt jene Tatsachen benennt, die die Angeklagten zu einem -Blockr, ciner Organisation von Staatsfeinden, erst zusammenfügen: einen Block bilden, eine Gruppe führen, Kräfte zusammenfassen, Kontakt aułnehmen, geheime Konferenzen abhalten und eine Plattform entwerfen. Solche Kategorisierungen beschreiben eher den arbeitsteiligen Prozeß in einer Bürokratie als den einer konspirativen Verbrecherorganisation. ${ }^{32}$

Wyschinskij, der die historische Bedeurung dieses Prozesses vor allem darin sieht, die Oppositionellen als organisierte Verbrecherbande zu entlarven, gelingt der Nachweis einer Organisation allein durch seine juristisch-dogmatiscbe Konstruktion der scrafrechelichen Teilnahme. Diese Konzeprion der Teilnahme hat er nach dem Moskauer Prozeß noch im Jahre 1938 in seinem Vortrag über die "Hauptaufgaben der Wissenschaft vom sozialistischen Sowjetrecht « erläurert: „Die vulgäre Vorstellung von der Teilnahme als einer Form der Vereinigung verbrecherischer Tärigkeit im engen Sinne dieses Wortes ist überlebt. Besonders jetzt, uncer den Bedingungen des Klassenwiderstandes der Welt der Ausbeuter gegen die siegreiche sozialistische Revolution hat sich das Problem der Teilnahme als einer Form des policischen Kampfes zugespitzt. «" Eine richtige Konzeprion für die juriscische Behandlung des Teilnehmerproblems könne man nur entwickeln, wenn man vom Charakter und den Besonderheiten des Klassenkamples in der heutigen Periode ausgehe. Da dieser Kampf gegen die sozialiscische Gesellschaft mmit größerer Schärfe, wie diese drei Jahre und die Prozesse gezeigt haben, die in diesen Jahren startgefunden haben ", ${ }^{34}$ geführt würde, müsse man die dogmatische Handhabung des Teilnahmeproblems an die besondere, eigenartige Organisationsform anpassen, in der der Klassenfeind aufgetreten sei. So könne der Begriff der Verschwörung oder des Komplotts auf den , Block der Rechten und Trorzkisten, nicht angewandr werden, da diese Einordnung "nichr nur eine direkte Vereinbarung zwischen den Beceiligten (. . .), sondern auch das Vorhandensein einer sogenannten wechselseirigen Anstiftung ${ }^{35}$ voraussenze und damit angesichıs der Verbrechen des 'Blocksı gerade den Grad der Gefährlichkeit der Haupsschuldigen und der aktivsten Verbrecher verwische. Auch ureffe der Begriff der Bande* nicht auf diesen $>$ Block $\mathrm{zu}$, da diese juristisch-dogmatische Einordnung mein subjektives Einverständnis der Komplicen über die Begehung einiger verbrecherischer Handlungen oder im Einverständnis über eine ständige oder dauemde verbrecherische Tärigkeiโ ${ }^{36}$ sowie das "Vorhandensein einer mehr oder weniger geschlossenen organisatorischen Form sowie die Grundsätze einer gewissen Hierarchie innerhalb der Bande voraussetze. Da all dies aber auf die Organisationsform des , Blocksı nichı zutreffen könne, gelangr Wyschinskij zu dem Schluß: Auf solche verbrecherischen Formationen wie den rechtstrotzkistischen Block können die alcen Begriffe der Verschwörung und der Bande vom juristischen Standpunkt aus kaum angewendet werden. ab7

Um aber gleichwohl zu einer strafrechtlichen Stigmatisierung zu gelangen, muß für Wyschinskij die »Teilnahme an einer Gruppe, die verbrecherische Handlungen begeht, die Verantwortichkeit eines Teilnehmers dieser Gruppe sogar dann nach sich ziehen, wenn er selbst nicht unmittelbar zu diesen verbrecherischen Aktionen

32 Siehe J. Carmichzel, a. a. O., S. 106 f.

33 A. J. Wyschinskij, Die Haupraufgaben der Wissersehaft vom sozialistischen Sowjetrechr, in: Sowjetische Beiträge zur Suazs. und Rechustheoric, a. a. O., S. 99.

34 Ebenda, S. 99 i.

is Ebenda, S. 101.

36 Ebenda.

17 Ebenda. 
in Beziehung stand und sein Einverständris zu ihver Begebung nicht erklärte. «\$8 Wenn nur ein Verbrechen aus der wallgemeinen verbrecherischen Haltung a 39 einer Gruppe hervorgehe, so sei schon die Teilnahme eines Teilnehmers im juristischen Sinne gegeben. Dabei sei eben nicht ein Kausalzusammenhang zwischen den Handlungen des Teilnehmers und dem Tateffolg der Organisation erforderlich, sondern lediglich ein Zusammenhang zwischen Individuum und kriminellem Gruppenzusammenhang überbaupt, der ein Endziel der verbrecherischen Guppentätigkeir irgendwie erleichtere oder vorherbestimme. Insofern sei sdie Teilnahme an einer derartigen Verbrechergruppe unabhängig davon, ob der in Frage kommende Teilnehmer an diesem oder jenem verbrecherischen Resultat teilhatte, durch das die verbrecherische Tärigkeit dieser Gruppe realisiert wurde. Das besagr, daß dieser Teilnebmer auch für dieses Resultat die Verantwortung ebenso wie für die ganze Tätigkeit seiner Gruppe cragen muß, gerade weil er ihr Teilnehmer ist. « ${ }^{40}$

Durch diese Tautologie gelingt Wyschinskij eine radikale Extension der surafrechtlichen Zugriffsmöglichkeiten. Wenn ein (politisches) Individuum nur in irgendeinem Zusammenhang mit anderen steht und diese wiederum mit einem *Verbrechen ", so ist es juristisch Teilnehmer dieser Tat und das fremde Verbrechen kann itum als eigenes zugerechnet werden. Auch eine Abwägung nach Schwere und Verantworrungsgrad an Handiungen muß nach dieser Konzeption des Organisacionsdelikts daran scheitern, daß jeder Beteiligte als Mittäzer an einer gemeinsamen Sache, der Wiederherstellung des Kapitalismus etwa, zumindest spirituell reilgenommen hat. $\mathrm{Daß}$ diese inhomogene, politisch konträre Parteirichtungen vertretende Schar von Angeklagien also als B Block der Rechren und Trotzkistena angeklagt werden konnte, gelingt durch die Definitions- und Zurechnungsmacht juristischer Dogmatib. Diese erst exlaubr es, eine Wechselbeziehung - soweit es die politischen Opposicionellen unter den Angeklagten betrifft - zwischen politischer Abweichung und strafrechrlichem Verratsvorwurf herzustellen. Auf der Basis einer juristischen Teilnahmelehre, die Beteiligung tautologisch begründet, muß die Zugehörigkeir zu einer politischen innerparteilichen Oppositionsgruppe, zu der auch nur eine sirafrechtlich verantwortliche Person zuzurechnen ist, notwendig als Teilnahme an einer Verbrecherorganisation erscheinen. Die politische Existenz der Angeklagten kann auf eine verbrecherische Existenz reduziert werden, Opposition gegen die herrschende Parteilinie wird zum sachverbaltlichen Anknüpfungspunkt für die Teilnahme am Verrat.

\section{Verbrechen durch Unterlassen \\ Die Ommissivdelikte im Moskauer Prozeß}

Ebenso gewaltsam wie die Zuordnurfg der Angeklagten zu einem "Block der Rechren und Trotzkisten " geschieht deren Zuordnung zu einer spezifischen kriminellen Handlung im Moskauer Prozeß durch die Ausweitung des Grundsatzes der Verantwortichkeit.

Strafrechtlich bedeutsam ist ein Handeln immer nur dann, wenn es von einer bescimmbaren Person nach engen Kausaliratsgesetzen bewirkt und final bewußt eingeleitet wird. Handeln kann aber strafrechtlich auch immer in einem Unterlassen bestehen. Ein strafrechclich bedeutsames Unterlassen allerdings erfordert eine

\footnotetext{
38 Ebenda, 5. 103

39 Ebenda.

40 Ebenda.
} 
Pflichten- oder Garantenposition des Unterlassenden zu einem Handeln. Indem nun im staliniscischen Strafrecht durch die Ausscrahlung des arbeits- und verfassungsrechrlichen Arbeitsgebotes jeder auf ein Handeln zugeschnittene gesetzliche Verbotstarbestand in einen Verpflichtungs- oder Geborstarbestand sich umwandelt, aus den Kommissivdelikten des Geserzbuches unter der Hand Ommissivdelikte werden, wird der Bereich der strafrechtlichen Verantwortbarkeit erweitert bis hin zu einer nbestimmten Einstellung zu den Pflichten der Sowjetbürgera." Nur mehr völlig an den Produktionsprozeß und seine verselbständigten Normen angepaßces Verhalten kann unter dem Zwang der Arbeitsverpflichtungen erlaubres Verhalten sein; jedes dysfunktionale Verhalten genügt nicht der Garantenstellung, in der der einzelne Arbeiter - und der offiziellen Definition nach gab es in der Sowjerunion in den dreißiger Jahren nur noch Arbeiter - durch seine Arbeitspflichr stehc, und \&ann strafrechtich poenalisiert werden.

Diese Entwicklung des sowjetischen Surafreches, durch die wie Arbeit schlecht-

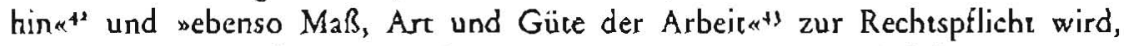
gelangt nun im Moskauer Prozeß in der Weise zur Anwendung, daß den Angeklagten, soweit sie eben als Volkskommissare, Ärzte oder Parteifunkrionäre Verantwortung trugen, jeweils in ihrem Verantwortungsbereich geschehene Pflichtverletzungen vorgeworfen werden, diese dann der politischen Orientieruog der ehemaligen parteiinternen Oppositionsgruppen final zugeordnet werden und schließlich als Momente einer politischen Verschwörung, kausal von den Verantwortlichen verursacht, final von der Opposicion gesteuert, enthüllt werden. Eine falsche Aussaat in der Landwirtschaft wird so im ersten Subsumtionsakc dieser strafrechrlichen Logik zu einer individuell zu verantwortenden Pflicheverletzung, diese wird im zweiten Subsumtionsakt zum strafrechtlichen Delikt der Schädlingstätigkeit, diese wird im dritten Akt der politischen Orientierung einer Oppositionsgruppe zugeordnet und daher zu einer politischen Tat. Als solche wird sie dann - auf die Wirkung der stalinissischen Teilnahmelehre hacte ich hingewiesen - geplante Saboragetärigkeic einer mir dem Ausland verbündeten - denn nur diesem kommt ein Sabocageakr zugure - und aut die Wiederherstellung des Kapiralismus zielenden Verbrecherorganisation. Nicht nur bezüglich der Schädlingstärigkeit legt die Anklage die Maßstäbe von Ommissivnormen an, auch der Mord an Kirow, nach Wyschinskij durch den , Block durch Unterlassen begangen, wird auf eine geplance Pflicheverlet\%ung: zurückgeführt.

Bevor der Mord an Kirow am I. Dezember 1934 geschah, war Nikolajew bereits einmal etwa ein halbes Jahr vor seiner Tat von der Sicherheitspolizei festgenommen und eine Schußwaffe bei ihm gefunden worden. Jagoda als Innenkommissar war für die Sicherheirspolizei und darrit mitrelbar für jeden Einzelakt ihrer Tätigkeit zuscändig. Nikolajew wurde seinerzeit, da ihm nichts nachzuweisen war, wieder auf freien Fuß gesetzt. Daran knüpft nun Wyschinskij im Prozeß an, rechner Jagoda diese Freilassung als Mord durch Uncerlassen zu. Dazu ein Ausschnir aus dem Prozeßprotokol:

Auf die Frage Wyschinskijs, was er unternommen habe, um den Mord an Kirow zu vollziehen, entwickelt sich folgender $»$ Dialog :

Wagoda: Ich erteilte eine Verfügung an Saporoskez. Als Nikolajew festgenommen wurde...

4 A. J. Wyschirskij, Fragen des Rechus und des Staates bei Marx, a. a. O., S. 39.

$42 \mathrm{~K}$. Westen, Die rechusuheorerischen und rechispolitischen Ansichten Josef Stalins, Konstanz 1959, S.

214.

4) Ebenda. S. 214 . 
Wyschinskij: Das erstemal?

Jagoda: Ja. Saporoskez kam her und berichtete mir, daß ein Mann festgenommen wurde...

Wyschinskij: In dessen Aktentasche?

Jagoda: Sich ein Revolver und ein Tagebuch befanden. Und er hat ihn auf freien Fuß gesetzi.

Wyschinskij: Und Sie hießen das gut?

Jagoda: Ich nahm das zur Kenntnis.

Wyschinskij: Und dann ereilten Sie die Weisung, der Ermordung von Sergej

Mirowitseh Kirow keine Hindernisse in den Weg zu legen?

Jagoda: Ja, ich erteilre... So war es niche.

Wyschinskij: In einer erwas anderen Fassung?

Jagoda: Es war nicht so, aber das ist ohne Belang.

Wyschinskj: Haben Sie eine Weisung gegeben?

Jagoda: Eine Bestärigung. \&4 $^{4}$

Der ehemalige Volkskommissar des Inneren hätte also, nach dieser strafrechtlichen Verpflichtungslogik, die Freilassung Nikolajews verhindern müssen. Er bestärigre aber die Enclassungsverfügung seiner Uncergebenen. Auffallen muß, wie weit hier der Kausalzusammenhang 7 wischen einem pflichtwidrigen Unterlassen und einem kriminellen Tarerfolg gezogen wird. Sicherlich war die Bestätigung Jagodas ein Glied in einer weiren Kerte von Kausalzusammenhängen, die zur Ermordung Kirows führte. Wäre Nikolajew festgehalten worden, hätte er Kirow nicht umbringen können. Dach liegt dieses Glied der Kausalkezte dem Törungsakt so fern und ist bei einer Betrachtung ex tunc so wenig kalkulierbar, daß Jagodas Bescärigung der Enclassung Nikolajews kaum Beziehung zur Törung Kirows hatre. Erst aus der post-festum-Betrachung, die die einzelnen Glieder einer Kausalketre rekonstruieren kann und für die sie dann gleichsam notwendig auf den Mord hinauslaufen muß, ist Jagodas Unterlassen kausal für die Törung Kirows.

Diese Verpflichtungsiogik des staliniscischen Strafrechts produziert zugleich notwendig die Umkehr der Beweislast: Dem pflichrwidrig Handelnden ist es aufgegeben, die Rechtmäßigkeit seines Handelns zu beweisen. Ex post ist das aber unnöglich. Die Resultate strafen ihn Lügen.

\section{Der strafrechtliche Manichäismus und die wirkliche Geschichte}

Die politischen Konsequenzen der Herrschafrslogik des Moskauer Prozesses springen ins Auge. Dazu ein Beispiel. Einer der Anklagepunkte gegen Bucharin besagre, in den Worcen Wyschinskijs,

„daß im Jahre 1918, unmitrelbar nach der Oktoberrevolution, in der Periode des Abschlusses des Brester Friedens, Bucharin und seine Gruppe der linken Kommunisten und Trorzki mit seiner Gruppe gemeinsam mit den sinken Sozialrevolutioaären eire Verschwörung gegen Lenin, als das Haupt der Sowjecregierung, organisierc haben. Wie aus den Materialien der Untersuchung ersichelich ist, verfolgten Bucharin und die anderen Verschwörer das Ziel, den Brester Frieden zu vereireln, die Sowjetregierung zu stürzen, Lenin, Stalin und Swerdlow zu verhaften und zu ermorden und eine neue Regierung aus Bucharinleuren, die sich damals zu Maskie-

44 Prozeßberichц, a. a. O., S. 410 I. 
rungszwecken linke Kommunisten nannten, sowie aus Trotzkisten und slinken Sozialrevolurionären zu bilden, «s

Daß es einen Plan zur Ermordung der Regierung gegeben habe, stritt Bucharin erbittert ab, wie sich aus dem Prozeßbericht ergibt:

„Wyschinskij: Ich tragte, hatren Sie einen Plan, im Jahre 1918 Genossen Stalin zu verbaften?

Bucharin: Nicht Stalin, sondern es anırde geplant, Lenin, Stalin und Swerdlow zu verhafien.

Wyschinskij: Also alle drei: Lenin, Salin und Swerdlow?

Bucharin: Ganz richig.

Wyschinskjj: Folglich niche Genossen Stalin, sondern die Genossen Stalin, Lenin und Swerdlow?

Bucharin: Ganz reche.

Wyschinskij: Bestand der Plan einer Verhafung?

Bucharin: Ich sage: es bestand kein Plan, sondern es fanden diesbezügliche Gespräche statt.

Wyschinskij: Auch über die Ermordung der Genossen Stalin, Lenin und Swerdlow? Bucharin: Keinesfalls. स $^{46}$

Obwohl Wyschinskij Bucharin noch mehrmals mil dem Mordvorwurf konfroncierte und sogar eine Reihe von bereits völlig in ihrer Identität gebrochenen Zeugen aufreten ließ, blieb Bucharin bei seiner Aussage. Er versuchte vielmehr, gegen Wyschinskijs Ordnungsrufe, den spezifischen Uncerschied zwischen politischen Orientierungen und Gesprächen im Jahre 1918 und einer juristischen Behandlung der innerparteilichen Kämple im Jahre 1938 zu verdeutlichen.

Die politischen Orientierungen der »linken Kommunisten $*$ um Bucharin im Jahre 1918 sahen vor, statr eines schmählichen und äußerst verlustreichen Friedens mir Deucschland den revolutionären Krieg fortzusetzen und in tiefgestaffelrem Parisanenkampf die geschwächte deutsche Armee zu demoralisieren, um die Revolurion ins Herz des Imperialismus zu tragen und das internacionalistische Versprechen der proletarischen Revolucion wahr zu machen."7 Lenins "Thesen über den sofortigen Abschluß eines annexionistischen Separatfriedens ${ }^{4^{8}}{ }^{8}$ bedeuteten für sie daher Verra an der Weltrevolution. Als dann der Friedensvertrag unterzeichnet, sie selber von den Anhängern Lenins nicht nur ihrer ursprünglichen Mehrheir in der Parzei für den Krieg, sondern auch offen aller parteiinternen Artikulationsmöglichkeiten beraubr worden waren, da spielten sie einen Augenblick mit dem Gedanken, die Spaltung hinzunehmen, die snationalistischer Regierung zu verhafren. Doch die Einheit der Partei siegre über die Prinzipien.99

Diese ungeheuer dramarische und politisch komplexe historische Situation wird nun im Moskauer Prozeß nach Maßgabe von juristisch-normativen Übertragungsregeln auf einen strafrechtlichen Vorwur reduziert. Die juristische Betrachrungsweise selektiert aus dem tarsächlichen, politisch gänzlich widersprüchlichen Geschehen eine simple Alternative von Schuld oder Unschuld heraus, konstruiert eine Verschwörung dorr, wo politischer Tagestampf statrfand, und verzerrt mit dieser

45 Prozeßberichi, a. a. O., 5. 701.

46 Eberda, S. 413

4) Siche dazu sun anderer R. V. Daniels, Das Gewissen der Revolution, Kommunistische Opposition in der Sowjerunion, Berlin 1978, S. $94 \mathrm{H}$.

${ }_{4}^{8}$ W. I. Lenin. Thesen über den Abschlvß cines annexioniscischen Scpararfriedens, in: ders, Ausgewählte Werke, Berlin 1970, S. 603 ff.

49 So R. V. Daniels, a. a. O., S. 105. 
Konstruktion das wirkliche Gewebe der Geschichte. Geschichtsfälschung ist das notwendige Resultar der juristischen Betrachtungsweise. In dem Maße nämlich, wie die jurisrische Klassifikationsweise des innerparteilichen Kampfes um den Friedensvertrag von Brest-Litowsk von 1918, innerhalb dessen die Gespräche um die Verhaftung der Regierung nur eine Episode blieben, zur herrschenden Interpretation in der Parreigeschichte der dreißiger Jahre avanciert, kann diese verzerrte und entstellte Interpretarion selber wieder als politisches Mittel gegen die ehemaligen Oppositionellen eingeserzt werden. Die Verfälschung ihrer Konzeprionen und Uberlegungen nach Maßgabe juristischer Ubertragungsropoi macht aus dem Kampf um die richtige Linie jeweils schon die Vorbereitung eines Verbrechens. Indern die Interpretation der Geschichte auf der Matrix strafrechtlicher Selektions- und Klassifikationskriterien zur herrschenden Geschichrsinterpretation wird, verstelle sich jede Möglichkeit, die Geschichre politisch und damit offen und zukunftsgerichter zu incerprecieren. Denn wo die polivische Interpretation Widersprüche analysieren könnte, da stellt die strafrechtlich-juristische nur hermetische Resulcate fest. Der Begriff von Geschichte als einer in der Zukunfesperspektive kontingenten, politisch zu bewältigenden Aufgabe wird von der poenalisierenden strafrechtlichen Betrachrung eliminiert. Geschichte hat stattgefunden, aber es gibt sie nicht mehr. Und in der Geschichte gab es nur Schuidige und Unschuldige: das beweist der Moskauer Prozeß.

Wyschinskij bezeichner die beiden Seiren dieses Manichäismus in seinem Plädoyer ganz deutlich:

"Gerade sie, diese Verbrechen, erklären (sic!) den wirklichen Lauf der Dinge, die wirkliche Logik der Ereignisse und des Kampts, die zwei Welten, zwei Blocks einander gegenüberstellten: den Block der Verräter, der Söldlinge des ausländischen Kapitals, der jetzt durch den Zorn und die Macht des großen Sowjetlandes entlarvt und zerschlagen ist, den Block der Verrärer, die mic ewiger Verachtung, ewiger Schmach und dem Fluch der Millionenmassen des werktätigen Volkes der ganzen Welt bedeckt sind - und den Block der Sowjetpatrioten, die in der Liebe zu ihrer Heimat groß und unbesiegbar sind, die mehr als eine historische Schlacht gegen die Feinde gewonnen haben, die unter der Leitung der Kommunistischen Partei und des großen Stalin bereit sind, jeden beliebigen Feind unter beliebigen Bedingungen zu beliebiger Zeit, wober er auch kommen möge, ungeachtet jeglichen Verrats, einen vemichtenden Schlag zu versetzen..* ${ }^{50}$

$E_{s}$ ist Bucharins Verdienst im Moskauer Prozeß, diese These des ehemaligen Menschewisten Wyschinskijs, die juristische Logik sei die wirkliche Logik der Geschichte gewesen, so weir als möglich durchbrochen zu haben. Dieses so weit als möglichs muß heißen, daß Bucharin die grundsätzliche Verantwortung für die ,konterrevolutionäre، Tätigkeit des,Blocks der Rechren und Trorzkisten< übernahm. Im Detail aber wies er den strafrechclichen Vorwurf der Spionage, der Vorbereitung von Terrormaßnahmen und des Aufstandes von sich. Der , Block der Rechten und Trotzkisten habe als Organisation gar nicht existiert, dena „die Mitglieder einer Räuberbande müssen einander kennen, um eine Bande zu sein und mireinander in mehr oder minder enger Verbindung stehen. Aber ich sah zum erstenmal in der Anklageschrift den Namen Scharangowitsch, und ihn seibst sah ich zum erstenmal vor Gericht. Zum erstenmal erfuhr ich von der Existenz Maximows. Niemals war ich mit Pletnjow bekannt, niemals war ich mit Kasakow bekannt, niemals sprach ich mir Rakowski über konterrevolucionäre Angelegenheiten, nic- 
mals sprach ich über diesen Gegenstand mir Rosengolz, niemals sprach ich darüber mir Selenski, niemals im Leben sprach ich mit Bulanow usw. \&s

Die Angeklagten sind nach Bucharin im juristischen Sinne keine Gruppe, und als Block hätten sie nicht existiert - es sei denn, ihre politischen Abweichungen als das ihnen partiell gemeinsame würden nach Maßgabe einer politischen Logik interpretiert, die Abweichung zu objektivem politischen Verrat erkläre.

Dieses Interpretationsangebor Bucharins an den Ankläger lehnte Wyschinskij aber ab; er beharre auf der juriscischen und damic geschichtszersiörenden Verbrechensversion.

\section{Das stalinistische Strafreche als Mittel repressiver Sozialgestaltung}

\section{Sowjetisches Strafrecht als Speerspitze staaclichen Verwaltungshandelns}

In dem Maße, wie sich in der Stalin-Zeit das neue, auf unmitcelbarer Herrschaft einer Staatsbürokracie gegründete System der Vergesellschaltung zunächst mic terroristischen Mitreln durchsetzte und sich schließlich konsolidierte, der Staat die Gesellschaft gleichsam in sich aufhob, veränderte sich das Verhältnis des gesellschaftlichen Úberbaus zur Basis der materiellen Produkrion insgesamt. Wie der Staat wohne vermittelnde Faktoren zur direkten politischen Organisation des Produkrionsprozesses, der allgemeine Manager der verstaatlichten Wirschaft $x^{\prime 2}$ wurde, verwischten sich die Dimensionen von Basis und Úberbau, wurde der Ubberbau jener Funktionen beraubr, die wgegenüber der Basis transzendent und antagonistisch sind.ess

Dieser Verlust der genuinen Uberbauqualitär des jurisrischen Normengefüges kommt in der stalinistischen Rechtstheorie zum Ausdruck. Das muß zunächst erstaunen, doch finden wir in A. J. Wyschinskijs Rechtscheorie trotz ihrer falschen begrifflichen Uberhöhungen, ihrer mechaniscischen Denkweise und ihrer ideologischen Verbrämungen eine scharfsinnige, mir Gespür für die Realität der sorvjecischen Rechtsentwicklung ausgeführte Analyse. Wyschinskij läßt in seiner Rechtstheorie die Marxsche Uberbaucheorie als analytisches Mirtel zur Darstellung der Beziehungen des Rechts zu den gesellschaftlichen Verhältnissen, die es bedingen, einfach fallen. Dies ist nicht nur Ideologie und damit falsches Bewußtsein; der Realprozeß, in dem das Recht seine Qualität als Uberbauphänomen verliert, kommt im rechrspositivistischen Denken Wyschinskijs zum Ausdruck. Seine Definition des Reches laucet:

"Das Recht ist die Gesamtheit der Verhalcensregeln, die den Willen der herrschenden Klasse ausdrücken und auf gesetzgeberischem Wege festgelegt sind, sowie der Gebräuche und Regeln des Gemeinschaftslebens, die von der Staatsgewalt sanktioniert sind. Die Anwendung dieser Regeln wird durch die Zwangsgewalt des Staates gewährleistet zwecks Sicherung, Festigung und Entwicklung der gesellschafrlichen Verhälınisse und Zustände, welche der herrschenden Klasse angenehm und vorteilhaft sind. «st

S1 Ebenda, S. 8361.

S2 H. Marcuse, Dic Gesellschuíslehrc des sowjetischen Marxismus, Darmstadt-Neuwied 1974, S. 120.

s3 Ebenda, S. 120. Vgl. auch: H. J. Krahl, Konstimuion und Klassenkampt. Zur historischen Dialekrik von bürgerlicher Emanzipation und prolezarischer Revolution, Frankfurd $M .197$ 1, S. 273 f; auch: exemplarisch an der Herausbildung eines dogmarisierzen Marxismus als Instinution O. Negt, Marxismus als Legitimationswrissenschafe. Zur Gencsc der stalinistischen Philosophie, in: Bucharin/Debarin, Kontroversen über dialektischen und mechanistischen Materialismus, FrankfurdM. 1974.

SA A. J. Wyschinskij, Die Huuptaufgaben der Wissenschaft vom sozialisuischen Sowjetrecht, 2. 2. O., S. 76. 
Zwar wird hier zur Erklärung der Genesis des Rechts noch auf gesellschaftliche Klassenbeziehungen, die sich im Recht ausdrücken, leginimatorisch verwiesen und die Gelung der Norminhalte mit der Sanktionswirkung staatlicher Gewalt in Beziehung geserzt. Die Formqualizät des Rechts dagegen wird nicht mehr als Problem gesehen.

Die Perpetuierung des Rechts als Regelungsform, wie sie realhistorisch in der etauscischen Gesellschaftsformation ihre Basis hat, bringt Wyschinskij gegenüber den sowjecischen Rechtstheoretikern, die noch in den zwanziger Jahren das Absterben des Rechts als Resultat einer sozialistischen Entwicklung gesehen hatcen, zum Ausdruck. Die materialen Rechtscheorien von Srücka", der als Justizkommissar und Oberster Richter der RSFSR zu Beginn der zwanziger Jahre in seiner Rechrstheorie an der Marxschen Theorie vom Absterben von Staat und Recht festgehalten, zugleich für die Übergangsperiode sich um die Gewinnung eines revolutionären Rechts des Proletariars bemüht hatce, und von Paschukanis ${ }^{36}$, der Recht allein als Produkr einer kapitalistischen Form der Gesellschaks begriffen und damit die Möglichkeit einer eigenständigen, ssozialistischen Rechrstheorie grundsäzzlich bestritten hatte, brandmarkce er als verbrecherische Verleumoungen, deren Sinn nur in der Schwächung des ssozialistischen< Seaaces und Rechces liegen könne. Der »schädliche antimarxistische Charakter a" der Theorie vom Absterben des Rechts zeige sich - so Wyschinskij - schon an deren praktischen Folgen; von ihr nführr ein direkter Weg zu der nihilistischen Einstellung zum Sowjetrecht als einem bürgerlichen Recht; zur Schwächung der Rechtsinstitute, die angeblich zum unverzüglichen Abscerben verdammt sind, und schließlich zur direkten Entwaffung des Proletariats, dem eine der schärfsten und mächtigsten Waffen im Kampf gegen die Feinde des Sozialismus aus der Hand geschlagen wird. ${ }^{38}$ Während gerade die faschiscische Bourgeoisie im Westen ohne juristische Verkehrsformen auskomme und das Recht in sein Gegenteil verkehre, nfestigt die sozialistische Gesellschaft der Arbeirer und Bauern in Gesezz mit Hilfe der ,juristischen Momenter ihre sozialistischen Eroberungen ${ }^{\prime}$, denn zur Umgestalrung der Gesellschaft brauche das Prolecariat "den Staat, braucht es einen Staatsapparat und eine bestimmte staatliche Ordnung - die sozialistische Rechtsordnung. Das bedeuter Beständigkeit der sozialistischen gesellschafclichen Verhältnisse, der sozialistischen Disziplin, Achtung vor den Regeln des sozialistischen Gemeinwesens, Achrung und Sicherung der Unantastbarkeit des gesellschaftlichen sozialistischen Eigentums als der Grundlage der gesamten Sowjecordnung, Einhalcung aller sowjetischen Geserze. ${ }^{60}$

Sieht man einmal von der simplen und objekcivistischen Identifizierung des Staates mir den proletarischen Inceressen ab, so erweist sich auch diese Kritik als Widerspiegeiung eines realhistorischen Prozesses der Gesellschafts- und Rechtsentwicklung. Denn in der Tat konnte Stučkas Theorie der revolutionären Gesetzlichkeit nur solange marerielle Wirksamkeir beanspruchen, wie sie im Rahmen der neuen Okonomischen Politik durch die großen zivilrechtlichen und strafrechtlichen Kodifikationen dieser Zeit Stabilität und Rechtssicherheit für den gesellschaftlichen Reorganisationsprozeß nach den Wirren des Bürgerkrieges und des Kriegskommu-

is P. Y. Stucka, Dice revolutionäre Rolle von Staat und Rechr, FrankfurcM. 1969.

s6 E. Paschukanis, Algemeine Rechislehre und Marxismus, Frankfurd/M. 1969.

s7 A. J. Wyschinskij, Fragen des Rechts und des Staares bei Marx, in: Sowjetische Beitrige zur Staats- und Rechsstheorie, Berlin 1953, 5. 38.

(8 Ebenda, S. 38.

ig A. J. Wyscbinskij, Zur Lage an der theorecischen Rechosiront, Moskau 193), in: Reich (HB.), Marxistische und sozialisische Rechtseheorie, FrankJun/M. 1972, S. 117.

60 Ders, Fragen des Reches und des States bei Marx, in: Sowjerische Beiträge zur Suats- und Rechtsiheo ric, Berlin 1973, 5. 18. 
nismus versprach und die staatlich kontrollierte Wiederherstellung des Kapitalismus in beträchtlichem Ausmaß* (Lenin) mit kapitalistischen Rechtsinstituten und Regelungsweisen begleitete. Auch die radikale Kritik des Rechts durch Paschukanis hatre realhistorisch nur solange die Bedeutung einer materiellen Gewalt, wie sein , Rechtsnihilismus in der Zeir von 1927 bis $193 \mathrm{r}$ dazu dienen konnte, die Nichtanwendung der bis dahin gelienden, die Privatrechtssubjekte vor willkürlichen Eingriffen schürzenden zivil- und strafrechtichen Normen während des administrativen Terrors bei der Kollekcivierung der Landwirtschaft, der Umstrukturierung der Industrie und der Enteignung der Kleinindustriellen dadurch zu legicimieren, daß sie das scheinbar rechtsmißachtende verwaltungsmäßige Vorgehen des Staates schon als eine UJberwindung des bürgerlichen Rechishorizontes erscheinen ließ. ${ }^{6 r} \mathrm{Nach}$ dem das neue System der sozialökonomischen Austauschbeziehungen sich konsolidiert hatte und der rechtlichen Versichenng bedurfe, mußte Paschukanis seinem -Rechtsnihilismusı abschwören. Im Jahre 193 I bezichrigte er, ganz im Einklang mit den neuen Anforderungen, die das System der etatiscischen Vergesellschaftung an das Rechtssystem stellte, seine eigene Theorie des Absterbens des Rechts mit dem Übergang zur sozialisrischen Gesellschaft einer Reihe wschwerer formaler Fehler a. ${ }^{6}$ Wie bestimmt nun Wyschinskij, für den das Sowjetrecht bereics als «sozialistisches Recht ${ }^{6}{ }^{6}$, dem SSozialismus` wesensmäßig zugehöriges Recht, zu gelten hat, die Funktion dieses sneuen Rechts? Für ihn ist alles Recht zunächst Staatsrecht, Mittel der staatlichen Verwalrungstäcigkeic. Es ist das Mittel des Sraates, des einzigen ihm vorstellbaren Rechessubjekts, bei der »Regelung der gesellschaftichen Verhältnissea $^{6_{4}}$ und damit - und dieses Beispiel aus der physikalischen Mechanik ist nicht zufällig - der "Hebel der Verwaltung "6s. Als Hebel der staatlichen Verwaltungstätigkeit und der etatisrischen Umgestaitung der Gesellschaft spielt es neine große schöpferische und organisatorische Rolle ${ }^{66}$. Als gänzlich auf die Erfordernisse der Staatstätigkeit abgesteltes Rechs gehen alle traditionellen Rechisgebiete, ihrer ursprünglichen materialen Regelungsmaterien gleichsam beraubt, im Staatsrecht auf. "Im weiteren Sinne umfaßt das Staatsrecht auch das Sirafrecht, das Verwaltungsrecht, das Zivilrecht, das Gerichtsrecht (ProzelBrecht) und das Arbeitsrecht. ${ }^{67}$ Wie auch das Strafrecht auf die Erfordernisse der Verwaltung zugeschnitten wird, zeigt schon Wyschinskijs Definition dieses Rechesgebieres. Bei ihm heißr es: „Das sowjetische Strafrechr ist Ausdruck und Regularor der gesellschaftichen Verhältnisse, die zwischen dem Sowjetstaat und den Sowjetbürgern in vom Staat (sic!) und im Namen des Staates (sic!) von der Staatsgewalt (sic!) geregelten Fällen sowie unter bestimmten, ebenfalls von der Staatsgewalt (sic!) festgelegten Umständen eatstehen. ${ }^{68}$

Ein Rechtsgebiet allerdings schiebt sich in Wyschinskijs Rechtscheorie gegenüber den anderen Teilgebiecen des Staatsrechtes in den Vordergrund. Das Verwaluungsrecht nimmi für ihn norwendig deshalb eine dominante Stellung innerhalb des Staatsrechtssystems ein, weil die staatiche Verwaltung unmittelbar die „Verwirklichung der Aufgaben der Dikratur des Proletariats, Realisierung des Willens des

61 Siehe D. Plaff, Die Entwickiung der sowjecischen Rechtslehre, a. a. O., S. $101 \mathrm{ff}$.

62 E. Pascbukanis, Für cine maxistisch-leninisuisebe St1ats- und Rechtstheorie, Moskau 1931, in: N. Reich ( $\mathrm{Hg}$ ), Maxiscische und sorialistische Rechischeorie, a.a.O., S. I11; siche auch in diesern Helt, S. ${ }_{402}$ if 63 A. J. Wyschinskij, Fragen des Rechts und des States bei Marx, a. a. O., S. I7.

64 Ebenda. S. is.

65 Ebenda

66 Ebenda, S. 17

67 A. J. Wyschinskij, Dic Haupraufgaben der Wissenschafr vom sozialisrischen Sowjertechl, a. 2. O., S. 82.

69 Ders., Fragen des Rechis und des Seaztes bei Marx, a. 2 O., S. 19. 
Sowjervolkes ${ }^{\text {(s) }}$ darscellt. Folglich muß das sowjerische Verwaltungsrecht »im System des Sowjetrechts eine der wichtigsten Stellen einnehmen . $^{7^{\circ}}$ Diese Funktionsbestimmung, die W/yschinskij mit einer Gewichrung von Regelungsgebieren innerhalb des sowjerischen Rechessystems verbindet, gewinnt in der Tat ihr fundamentum in re durch die Modifizierung dex materialen Grundlagen des Sowjetrechrs in der Stalin-Zeit.

Mit den Getreideerfassungsmaßnahmen in der Versorgungskrise im Winter 1927/28, der Kollekcivierung der Landwirtschaft und der Zwangsindustrialisierung wurde die privatrechelich geregelte Form der Vergesellschafrung, die mit der Periode der Neuen Okonomischen Politik wesensmäßig verbunden war, in den Hintergrund gedrängt. Das Privatrecht trat seine dominante Stellung an das Verwaltungsrecht ab, wurde - seiner materiajen Geltungsgrundlage beraubt - peripher, starb àso in dem Maße, wie es seine organisierende Kraft über die ökonomischen Austauschprozesse verlor, gleichsam ab. Die gesellschaftlichen Bedingungen für diese neue Dominanz des Verwaltungsrechts (Kollektivierung, Planökonomie) wurden bis 1934 in Enteignungsverfahren durch stratrecheliche, ansonsten aber durch Verwaltungsakte, nicht-rechtlich geregelte administrative Eingriffe, geschaffen.

Die verwaltungsrechtlichen Regelungen, wie sie ir den Fünf-Jahr-Plänen sich zwar monetärer Kalkularions- und Berechnungsmethoden bedienten, den gesellschaftlichen Produktionsprozeß aber nach Gebrauchs- und Nutzeffekren organisierten, wurden zum zentralen staaclichen Gestalcungsmittel. Klaus Westen hat die gestaltende und sschöpferischer Qualität dieses Sowjetrechrs, die auch in Wyschinskijs Rechtstheorie zum Ausdruck kommt, wie ich gezeigt hatre, nur mit Veruunderung registrieren können. „Nunmehr wird das Gesetz zur treibenden Kraft der Gesellschaft, und es wird dem positiven Recht erstmalig die Funktion übertragen, die Lebensverhältenisse niche nur zu fixieren, sondem erscmals konsticutiv zu regehn. $x^{7}$ Damit werde - so Westen weiter - das sformale Recht zur eigentlichen schöpferischen Kraft der sowjetischen Gesellschaft ... . ${ }^{72}$ In der Tat wird es als Wirtschattsverwaltungsrecht unmittelbar zur ökonomischen Potenz, strukturiert als Organisationswissen den stofflich-energetischen Arbeitsprozeß, ohne daß der ökonomische Mitteilungsmechanismus des Marktes dazwischentrirt.

Als Recht hat es seine Existenz neben den gesellschaftlichen (Hand-)Arbeitsprozessen, geht nicht als Regelung des gewohnheirsmäßigen Zusammenlebens der Menschen in diesen auf, indem es nur sachliche Verhältnisse zum Gegenstand härte, sondern organisiert diese Arbeitsprozesse erst als arbeitsreilig-gesellschaftliche und zwingr sie damit den Menschen als den Objekten seines vergesellschaftenden $\mathrm{Zwanges}$ auf. Insofern ist es als gesondertes Normensystem auch Juristen- oder doclı Fachleurerecht, seine Durchsetzung ist institutionell verselbständigt und - abgesehen von der unteren Gerichtsbarkeir - gänzlich professionalisierr. Seine Sätze sind nach internen Regeln der Kohärenz, Operationabilität und instrumenteller Rarionaliäri systematisiert. Als konventionelles Recht drückt es wie das bürgerliche.

69 Ders, Die Hauprautgaben der Wissenschaft vom sozialiscischen Sowjetrecht, a. a. O., S. 83 .

70 Ebenda, 5.83 .

7I K. Westen, a. a. O., S. 39 f.

72 Ebenda, S. 60. Das Verwaltungsrechr mit seiner sinkknrellen Gewalibexiehung zu den unter seine Rcgelungsmaterien subsumierren Individuen bringt Vergesellschalung und damir auch die spezifische Arbeitsucilung erst hervor. Die verwaliungsmäßige Assoziationsform ist das Surrogat einer Ireien Assoziation der gesellschálichen Individuen. Vol, zu diesem Gedanken J. Perels, Der stazilich verordnese Sozislismus. Thesen zur Verfassungstheorie der Sowjetunion, in: Roulcurhner $\left(\mathrm{H}_{\mathbf{b}}\right)$, Probleme der marxiscischen Rechestheorie, Frankfurr/M. 1975, S. 145; zur Siellung des Verwalungsrechis in sowjecischen Rechessy'stems s. den zur Entwicklung des sowjecischen Verwalungsrechics noch immer maßgcbenden Aufsazz von N. Karadse-Iskrow, Das Verwalnungsreche in der Sowjetunion (Rußland) seir $1917,5.234$, der im Jahrbuch des öff. Rechıs، Bd. 23, im Jahre 1936 crschien. 
Privatrecbt «den Willen eines souveränen Gesetzgebers aus, der mit rechtichen Organisationsmitteln soziale Tatbestände konventionell regelt. 73 Wenn es also auch den Horizont des Rechts nicht verläßt, so bricht es doch mit der Tradition, der Typologie des bürgerlichen Privatrechts. Anders als dieser genuine bürgerliche Rechtstypus nämlich, der nur negierend Grenzen von prinzipiell unbegrenzten subjekuiven Handlungsberechrigungen kennr, erhebr es positive Ansprüche an materiales Handeln, regelt konkrete Produktionspflichten und -gebote. Auch regelt es niche universalistisch den ökonomischen Verkehr der Gesellschaft dadurch, daß es Warenbesitzern die Durchsetzungsformen ihrer Interessen zur Verfügung stellt, sondern es enchäls eine Vielfait konkretester Regelungen, die auf Erfordernisse der materiellen Produktion unmittelbar abgestellt sind und konkrete Arbeitsschritte, Stückzahlen, Qualitätsnormen unmittelbar als Rechtspflichten enchalten.

$\mathrm{Daß}$ alle anderen Rechrsgebiete des sowjerischen Rechtssystems an diesem dominancen Rechtrypus sich funktional ausrichten, konnte schon aus Wyschinskijs Theorie des Staatsrechts geschlossen werden. Das Strafrecht, dessen neue Funktionen hier interessieren, lehnt sich in doppelter Hinsicht an das Verwaltungsrecht an. Zum einen wird es selber Teil des Vervaltungskontrollrechtes. Das Verwaltungskontrollsystem in der Sowjerunion kannte nach dem Geserz über die Sowjeckontrollkommission von 1934 drei Kontrollinstanzen. Neben der staatlichen Kommission für Sowjetkontrolle, die alle Prozesse der maceriellen Produktion zum Gegenstand harte und der jeweils einer Verwairungsstufe übergeordneten Kontrolistufe bildete die Staatsanwaltschaft die dritte sowjetische Verwaltungskontrollinstanz." Kam den ersteren Instanzen die Aufgabe zu, durch Verfügungen unmitrelbar einzugreifen und disziplinarisch gegen funktionsunwillige Verwalunngsobjekte vorzugehen, so oblag dem strafrechelichen Zugriff der Staatsanwaltschaft die generalpräventive Ahndung von verwaltungsinternen Dysfunktionalitäten. Auf diesem Hintergrund wird auch der bereits erörterte Ubergang zu einem Verpflichrungsstrafrecht verständlich. Das Objekt der staatsanwaltlichen Verwaltungskoncrolle ist jeweils der konkrete auf Aufgaben verpflichtere Verwaltungs->Arbeiterc. Seine Bestrafung beruht sachverhaldlich daher auf dem Unterlassen von Handlungsptlichten. Die Ausdehung des Bereiches der strafrechtlichen Verantwortung und die Wiederautnahme der Surafzumessungsregel der ,Schuld, im staliniscischen Strafrechr"s sind unmittelbarer Ausfluß der Verwaltungskontrollärigkeit der Staatsanwaltschaft aut strafrechtlicher Grundlage. Schon in dieser Kontrollfunktion bildet das Strafrecht die eigentliche, mit absoluter Sankcionsgewalt ausgestattere Speerspitze der staaclichen Verwaltungskontrolle.

Strafrechtliche Eingriffe sind weiter dort erforderlich, wo noch immer Verkehrsformen vorherrschen, die der staatlichen $Z$ wangsvergesellschafrung strukturell entgegenstehen und wo Konflikepotentiale aufbrechen, die durch $\mathrm{Zwang}$ auf Dauer allein nicht zu befrieden sind. Sie durch punktuelle oder systematische Eingriffe zu demoralisieren und zu destrukturieren, ist die Funktion des Strafrechts außerhalb seiner Verwaltungskontrollfunktion. Das Strafrecht wird - um in Wyschinskijs

73 J. Habermas, Uberlegungen zum evolutionaren Stellenwert des modernen Rechis, in: ders., Zur Rekonseruktion des historischen Materialismus, FrankfurJ/M. 1976, S. 264. Wihtend in der NEP-Periode in der SU noch mehrere insriutionen als Legislative auferaten und Geserze verbindlich beschlossen, aunde ab 1936 nur noch der Rat der Volkskommissare als Legislacive anerkannt. Insoweil aurde Souveränicür als Einheit des Stateswillens wiederhergestellt.

74 Vgl. N. Karadse-1skrow, a. 2. O., S. 190 I., zu den umlangreichen Eingriffsbefugnissen und Aulgabenbereichen der sowjerischen Stazisanwaleschaf auch Westen, Die Kommuniscische Parce der Sowjecunion und der Sowjetsuazt, Köln 1968, S. 199 ff.

75 Siehe A. J. Wyschinskijs Austührungen zum Problem der Schulde, in: Die Haupuafgaben der Wissenschale vom sozialisuschen Sowjerrecht, a. A. O., S. $97 \mathrm{f}$. 
Sprachregelung zu bleiben - eine "mächtige Waffe ${ }^{76}$ des staaclich-bürokracischen ,Absolutismusı. Es gestaltet dessen unbeschränkte sozialökonomische Hegemonie.

\section{Der politische Prozeß als Mittel zur Durchsetzung der stalinistischen Herrschaftsstruktur}

Diese Gescaltungsfunktion des scalinistischen Strafrechts bestimmt den Moskauer Prozeß von 1938 in doppelter Hinsicht. Einerseits, und dies ist die nideologische Rationale « $\pi$ des Prozesses, gestalter er gekoppelt an die ihn begleitende Propagandakampagne ein Massenbewußtsein, in dem die strafrechtich verzerrte Interpretation von Geschichte zur materiellen Produktivkraft wird. Mit der Poenalisierung der prominenten Angeklagten, die die Revolution zu ihrem Beruf gemacht und bis zu ihrer Liquidierung nach dem Prozeß das "Gewissen der Revolution ${ }^{78}$ repräsentiert hatten, greift die Anklage nämlich in versteller Form reale gesellschaftliche Probleme der dreißiger Jahre in der Sowjetunion auf. Verborgen unter der individualisierten Form, in der sie im Prozeß als Schädlingstärigkeit, Saborage und Separacismus auftreten, offenbaren sich hier gesellschaftlich bedingte Dysfunktionalitäten, die gerade deshalb Gegenstände juriscischer Beurceilung werden, weil sie politisch tabuisiert und verdrängt worden warea.

Die Eindimensionalisierung des Massenbewußrseins hin auf das eine Ziel der linearen Entwicklung der technologischen Produkrivkräfte betrifft im Prozeß einmal das Problem der sozialökonomischen Beziehung von Stadr und Land, wie sie historisch gerade mit der $\mathrm{Zwangskollektivierung} \mathrm{verwaltungsmäßig} \mathrm{eingebunden}$ worden war. Nur auf der Grundlage der Zwangskollektivierung, des durch sie ausgelösren Widerstandes der Landbevölkerung und der of kuriosen landwircschaftlichen Fehlplanungen ${ }^{\prime y}$ sind die gegen den ,Block der Rechten und Trotzkistenc im Moskauer Prozeß aufgeworfenen Anklagepunkre, sie hären in der Landwirschaft Saborage, Diversion und Schädlingstötigkeit geleistet, zu verstehen.

Die ehemaligen Vertreter der sog. Rechren, Rykow und Bucharin, zuvor schon politisch der Kulakenfreundlichkeit bezichrigt, werden nun strafrechtlich zu Agenten der kulakischen Saborage auf dem Lande erklärt. Wann immer in der Landwirtschaft Krisen auftresen, so sind sie nicht systembedingt, sondern auf kulakentreundliche Kräfte in der Partei zurückzuführen. ${ }^{80}$ Das policische Beharren der BucharinFraktion auf der smyıschka-Konzepuion, auf dem Bünanis zwischen Arbeiterklasse und Bauern, und der linken Frakrion auf der Prämisse, daß die Kollektivierung möglichst mit den Bavern und gewaltlos vonstatten gehen sollte, macht diese Parteifraktionen zu Kollaborateuren des kulakischen Feindes, die ebenso strafrechtLich zu verfolgen sind, wie schon die Kulaken nach Art. 107 des Strafgesetzbuches verfolgt worden waren. ${ }^{81}$ Die strafrechiliche Logik, die im Moskauer Prozeß

76 A. J. Wyschinskij, Zur Lage der theorerischen Rechesiront, in: N. Reich (Hg.), Marxistische und sozialistische Rechestheoric, FrankfurdM. 1972, S. IY3.

77 P. Schulze, Herrschafe und Klassen in der Sowjeruaion. Die hiscorischen Bedingungen des Stalinismus, Frankfurt/M.-New York 1977. S. 187.

78 Sa die Bezeichnung von R. V. Daniels, D2s Gewissen der Revolution, Kornmunistische Opposition in der Sowjennion. Berlin 1978. 5. 473, für die bis heute nicht rehabilivienten opposivionellen Kommunisten in der Sowjerunion.

79 Vgl. R. Lorenz, Sozialgeschichte der Sowjerunion 1, 1917-1945, Frankfurd M. 1976, S. 200 ff.

so Vgl. zur sozialen Psychologie der Entkulakisienung R. Lorenz, a. a. O., S. 193 ff.

8s $\mathrm{Zu}$ den Methoden der Kollekuivierung vgl. R. Medwedjew, a. a. O., S. $10_{4} \mathrm{ff}$. 
eingeschlagen wird, beginnt da, wo die politischen Konzeptionen der Angeklagren in bezug auf die Agrarfrage sich niche durchseczen konnten. Weil sie als politische Konzepteure nicht erfolgreich waren, stehen sie als Vertreter des Widerstandes der Agrarbevölkerung gegen die stalinsche Gewaltpolitik vor den Schranken des Gerichts. Strafrechtlich sind sie damit die eigentlichen Autoren des Krisenzusammenhangs Landwirtschaft. Das Dilemma, das durch die stalinistische Zwangskollektivierung auf dem Lande angerichter wurde, wird so auf die politischen Gegner dieser Agrarpolitik abgewälzt. ${ }^{82}$ Die Wirren, die auf dem Lande herrschten, werden strafrechrlich als Verbrechen der Oppositionellen poenalisiert. Der Angeklagte Selenski schildert in seiner Selbstanklage einige dieser Wirren: Aufgrund der Direktive, in Mittelasien im Jahre 1929 die Kollektivierung der Landwirtschaft zu beschleunigen, - so Selenski - marbeiteten die lokalen Organisationen einen Plan beschleunigten Tempos der Kollektivienung aus und traten an die Organisierung von Kollektivwirtschaften heran, wobei sie von administrativen Methoden Gebrauch machten. Diese Methode des Kollektivwirtschaftsaufbaus wurde von national-chauvinistischen Organisationen aufgegriffen, die eine Reihe von Übergriffen organisierten. Als die Unzufriedenheit ein berrächcliches Ausmaß angenommen hatte und es nicht mehr möglich war, diese bei der Kollektivierung begangenen groben Fehter vor dem ZK zu verbergen, gab ich die Anweisung, man solle, anstact die Fehler zu korrigieren, es so einrichten, daß die Leute aus den Kollekrivwirtschaften austreten, und daß man das niche verhindern soll. Unter dieser scheinbar legalen Formel öffneten wir Tür und Tor für die Kulakenagitation und die Kulakenakcivicät, die auf den Zerfall der Kollekrivwirschaften hinausliefen. $x^{8_{3}}$

Was Selenski hier als Akt der Schädlingstärigkeit, begangen durch den , Block der Rechten und Trotzkisten schildert, ignoriert, daß die gesamte Kollektivierung mit madministrativen Methoden hergestellt wurde, daß die "Ưbergriffe a systematische Folge dieser Methoden waren, daß die wUnzufriedenheic nocwendig aus den systematischen "Ubergriffen« resultierte.

Ein weiterer politischer Hintergrund der Anklagen im Moskauer Prozeß ist in der gewaltsamen Industrialisienung zu sehen, die die staliniscische Parteiführung ab 1929 einschlug. Auch deren z. T. dystunkrionale und für große Teile der sowjerischen Arbeiterschaft katastrophalen Folgen wurden im Prozeß den Oppositionellen a's Verbrechen strafrechtlich zugerechnet. Im Prozeß erscheinen Verlangsamungen und Einschränkungen in der Großbautätigkeit ${ }^{84}$, Stockungen in $\mathrm{Handel}^{83}$, Qualitätseinbußen bei Nahrungsmitzeln ${ }^{86}$ und andere dem Zentralplan widersprechende ökonomische Entwicklungen ais von Agenten des kapitaliscischen Auslandes geplante Verbrechen. Auf die Beschreibung der tatsächlichen Dysfunkrionalitäten im Industrialisierungsprozeß folgt im Moskauer Prozeß jeweils die juristische Fikrion, daß man alle Probleme den Angeklagten zuschreiben könne. "Was von den Angeklageen gestanden wurde, bildete jeweils das im voraus amtlich präparierte Zerrbild der Wirklichkeit, ihre synthetisch bergestellte Alternativgestalt. Die Anklagebehörde präsentierte ein Sammelsurium von Einzelangaben, in denen sich wirkliches Geschehen unentwirrbar mit erdichtetem verflocht. $a^{87}$

Die juristisch serdichtece Schuldzureilung bewirkte durch ihre Verzerrungen eine Entlastung der politischen Führung von der Verantwortung für das Dilemma, das

82 Vgl. zusammentassend in A. J. Wyschinskijs Schlußplädoyer im Prozeßberichr, a. a. O., S. 730 ff.

83 Prozeßbericht, a. a. O., S. 357.

84 ProzeBbericht, a. a. O., S. 87 .

83 Ebeoda, S. 348 .

86 Ebenda, S. 361.

87 O. Kinchheimer, Politische Justiz, a. a. O., S. 167. 
ibre voluntaristische und keineswegs historisch notwendige ${ }^{88}$ Planungspolitik angerichtet hatte.

Den wohl umfassendsten Raum im Protokoli des Moskauer Prozesses nimmt die 'Beweisführung für den Vorwurf des politischen Separatismus ein. In Wyschinskijs Anklagerede wird dem >Block der Rechten und Trotzkisten s speziell zur Last gelegt, er habe sdie Zerstückelung der UdSSR und die Lostrennung der Ukraine, Belorußlands, der Mitcelasiacischen Republiken, Georgiens, Armeniens, Aserbaidshans und des Fernöstlichen Küstengebietes von der UdSSR ${ }^{89}$ zum Ziel gehabr und sei insofern zugleich eine Organisation »der bürgerlichen Nationalissen ${ }^{9 \circ}$ aus allen Randnationen der UdSSR.

Der politische Hintergrund dieses Anklagepunkres ist die Nationalitätenfrage, die durch die stalinsche Kollektivierungs- und Industrialisierungspolirik in den dreißiger Jahren sich als politisches Problem erneut stellte. Denn diese Politik, die mit systematischer scrafrechtlicher Verfolgung der Kulaken und administrativer Zusammenfassung der privackapitalistisch orientierten Bauern vor allem die überwiegend agrarisch strukturierten nichtrussischen Nationalitäten traf und zu den Gewaltmethoden des Kriegskommunismus zurückfand, lieferte dem latenten politischen Nationalismus die ökonomische Basis. «Die Härten der Kollekrivierung, die steigend zentralisierte Kontrolle, die Moskau im Interesse der Industrieplanung verhängre, und die allgemeinen Entbehrungen, die das Ergebnis der neuen Polirik waren, führeen unvermeidlich zu einem Wiederaufleben nationaler und antirussischer Empfindungen bei vielen der örtichen Kommunisten, die bereit gewesen waren, unter den leichteren Bedingungen der NEP Moskaus Führung hinzunehmen..*9' Nicht nur reakrionäre, ihre agrarkapitalistischen Privilegien verteidigende Großgrundbesitzer, sondern weite Teile der bäuerlichen Bevölkerung und nationalgesinnte Bolschewiki, die sich auf das Agrarprogramm der Rechten um Bucharin stützen konnten, wehrten sich zunächst gegen die Kollektivierung. Durch gewaltige Säuberungen in den nationalen bolschewiscischen Parteisckcionen und adminiscrative Repressalien, die, wie Lorenz anmerkt ${ }^{92}$, besonders die Bauern in der Ukraine und im Nordkaukasus trafen und in den Hauprgetreideanbaugebieren, dem Wolgagebiet, zu einer Flucht in die Kollektive führte, konnte erreicht werden, daß - wie die XVII. Parteikonferenz vom 4. 2. I932 feststellte - der Sozialismus auf dem Lande nun voliständig und unwiderruflich gesiegt hatte.

In dem $\mathrm{Maße}$, in dem mir der volkswirtschaftlichen Gesamtplanung die narionale Wirtschaftsentwicklung in eine Arbeissteilung eingefügt wurde, die die gesamte Sowjecunion berral und die die Nacionen auch ökonomisch völlig in ihrer Versorgung von dem russischen Zentralgebiet abhängig machte, wuchs der Widerstand der auf ihre nacionale Identitüt bedachten Bolschewiki. Durch Planungsvorgaben und, soweit möglich, durch Verzögerung der Einbeztung nationaler ökonomischer Ressourcen in die gesamtsowjetische Arbeitsteilung suchten sie die ökonomische Unabhängigkeit und damit die Fähigkeit ührer Nation, sich selber autonom zu versorgen, aufrechtzuerhalten. Solcbe Tätigkeit nun, Obstrukcion gegen die Kollcktivierung und Verzögerung der ökonomischen Verzahnung mit der Gesamtunion aus nacionalen Motiven, ist der Hintergrund des Separatismus-Vorourfs im Mos-

88 Insofern ist R. Lonenz, Sozialgeschichte der Sowjetunion, 1917-1949, FrankfurvM. 1976, S. 116 ff. zuxussimmen. Die Indusurialisierungspolitik wridersprach nicht nur den grundlegenden Erkenatnissen der Induscrialisienungsdiskussion; sic läßt sich auch nicht mit außerpolitischen oder mit ökonomischen Argumenten rechtferigen, wie das heute von sowjecischer Seite immer wieder versuche wird.* 89 Prozelbericht, a. 2 O., S. s.

90 Ebenda, S. 6.

91 L. Shapiro, Geschichce der KJdSU, Berlin 1962, S. jo1.

92 R. Lorenz, Sozialgeschichre, a. 2. O., S. 197. 
kauer Prozeß. Gerade die Gebiete mit noch bis in die dreißiger Jahre hinein funkrionierender tradicionaler Landwirtschaft werden im Moskaver Prozeß als gleichsam separatistische Krisengebiete genannt. Der Angeklagte Chodskajew geht in seiner Selbstanklage auf den hier erörterten Zusammenhang ein:

-Ich weiß nicht, ob dem Gericht bekannt ist, daß bei den bürgerlichen Nationalisten besonders in Mitrelasien eine solche Theorie bestand - eine geschlossene Wirtschaft zu organisieren, d.h. es so einzurichten, daß sich die Wirtschaft der Republik unabhängig von den anderen Teilen der Union entwickle, daß die Republik nach Möglichkeit so leben soll, daß sie für den Fall irgendwelcher Möglichkeiten zum aktiven, unmittelbaren Kampf die übrige Union nicht braucht. Und diese Theorie haben wir in die Tat umgesetzt, d. h. wir haben einen ersten Fünfjahrplan aufgestellt, der den Direktiven (sic!) der Union zuwiderlief und der Einstellung der Narionalisten auf eine geschlossene Wirtschaft entsprach. Wir planten die Wirtschaft so ( . . ), daß wir uns nach Durchführung dieses ersten Fünfjahresplans in wirsscbaftlicher Hinsicht von Sowjetrußland, von der Sowjetunion unabhängiger als jemals erweisen. Dieser Plan wurde in Moskau durchschaut. "93

Die ratsächlichen Anknüpfungspunkte der Anklage können hier nur exemplarisch erörter werden. Zusammenfassend produziert der Prozeß ein durch Individualisierungen und politische Schuldattributionen verzerrtes Bewußtsein von realen Problemen der sowjerischen Gesellschaft der dreißiger Jahre. In dem Maße, wie diese Form der Verarbeitung der Agrar-, Induscrialisierungs- oder Nationalitätenprobleme zum politischen Massenbewußrsein wird, stellen sich diese Probleme für das einzelne politische Subjekt nur mehr als Probleme der individuellen Leisrungsmot;vation, der Gewissenhaftigkeit in der Befolgung von Direkiven und der politischen Loyalizär, letzrendlich als Probleme einer erfolgreichen Internalisierung der Normen des entremóeten Arbeitsprozesses.

Uber diese Gescaltung von Massenbewußtsein hinaus aber, und djes ist die zweite, materiale Gestaltungsfunktion des Moskauer Prozesses, leistet die politische Justiz der dreißiger Jahre insgesamt mit der Liquidierung der alten bolschewiscischen Garde einen Beitrag zur Konsciturion der bis heute nur modifizierten Herrschattsstruktur in der Sowjetunion.

Von 1934 an waren nicht mehr bürgerliche Spezialisten Gegenstand von Strafverfahren, sondem die sowjerische Führungsschicht in der Partei und der staarlichen Verwalrung selber. Eine ganze hegemoniale Schicht wurde bis 1939 ausgetauscht. Von 1933 bis 1936 allein nahm, um einige Zahlen zu nennen, durch die Strafjustiz und die Säuberungen die Anzahl der Parteimiglieder und -kandidaten von dreieinhalb auf etwa zwej Millionen ab, die Spitze der Partei wurde bis auf einige wenige Mitglieder des Politbüros insgesamt liquidiert oder beging prophylaktisch Selbstmord. "Unter den 1827 Delegierten des XVII. Parteitages befanden sich lediglich noch 35 Delegierte, die die Ereignisse nach dem XVIII. Pareicag überlebe hatten

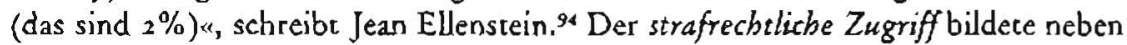
dem der politischen Verwalung das entscheidende Mittel, die radikale personelle Umstrukturierung aller Leitungstätigkeiten zu bewirken. Insofern gestaltete er unmictelbar die gesellschaftlichen Verhältnisse.

Im Gegensacz zu Withelm Ziehr, der in den Angeklagten der politischen Justiz der dreißiger Jahre keine nfestumrissene soziale Schicht ${ }^{95}$ mehr entdecken zu können

93 ProzeBbericht, a. a. O., S. 244

94 J. Elleinstein. Geschichte des ,Stalinismus،, Berlin 1977, S. 122. Der XVIY. Parteiug fand im Februar 1934, der XVIII. Parteicag fand im März 1939 start. Vgl zur Liquidationspraxis auch R. V. Danicls. 3. a. O., S. $43^{8}$, wnd R. Medwedjew, 2. a. O., S. 21, H. is W. Ziehr, a. a. O., S. 318 . 
glaubte und in ihnen nur den notwendigen Katalysator ${ }^{96}$ fir allgemeine politische Loyalitäts- und Wachsamkeitsappelle sah, hat Peter Schulze die Strafprozesse in einen systematischen Zusammenhang mit dem klassenmäßig differenzierten "Konstitutionsprozeß der Herrschaftsverhältrisse «7 der Stalin-Zeit gerückt. Für ihn sind diese Prozesse Teilvorgang ciner Konstitucion von Klassenherrschaft in der Sowjetunjon, die aus der Verschmelzung der noch während der NEP-Periode gegeneinanderstehenden Produktionsincelligenz und Legitimacionsintelligenz resultiert. Die Produktionsintelligenz, die als Betriebsleiter und Betriebskontrolleure in direkter Beziehung zu den Produktionsmitteln stand und sich weitgehend aus bürgerlichen sspezy', aber auch aus mit Leirungsfunktionen alimentierten alten Bolschewiki und sHelden des Bürgerkriegsı rekrutierte, war der Legirimationsincelligenz in der Partei und der Staacsspicze ihrer Funktion wie ihrer sozialen Herrschaftsstellung nach während der NEP untergeordner. Die Stalinsche Industrialisierungs- und Planungspolitik begnügte sich nicht damit, ihr die operative Selbständigkeit der Tätigkeit von Quasi-Kapicalisten zu nehmen und ibre Sonderinceressen, die sich während der NEP herausgebildet haten, in der Realisierung zu beschneiden. Bis 1934 wurden die bürgerlichen Spezialisten encmachtet und exemplarisch mit strafrechelichen Mirteln ihrer Funktionen beraubr. Ab 1931 wurde zugleich in einer abrupt einsetzenden Mobilisierungskampagne eine neue, sozialistische Produkrionsintelligenz ausgebildet, die aber an speziellen Parceischulen als Techniker, Ingenieure, Agronomen usw. geschulte Kader zugleich in ihrer politischen Orientierung an der Parteilinie der forcierten Industrialisienung ausgerichtet war..$^{98}$ Diese neve Intelligenz ersetzte zunächst die bürgerlicben Spezialisten, ihr gelang es aber zugleich, als loyale Stalinisten sehr schnell in der Partei Fuß zu fassen und dadurch die alte Unterordnung der Produkcionsinteligenz unter die bolschewiscischen Erben des historischen Auftrags der Oktoberrevolution aufzubrechen. Da sie entsprechend ihrer Stellung im Produkcionsprozeß und ihrer Induscrialisierungsaufgabe ideologisch die Industrialisierung mit dem Sozialismus idenrifizierte, koinzidierte sie mir jenem Teil der Legitimationsintelligenz, der sich gerade politisch gegen die Verfechter einer Fortsetzung der NEP oder einer moderateren Industrialisierung durchgesetzt harte. $\$$ Wie auch der Richtungswechsel in der politischen Justiz ab 1934 deuclich werden läßc, begann nun der personelle Austausch jenes Teils der Produktionsintelligenz nebsc ihres Anhangs in der Legitimationsintelligenz, der sich aus der revolutionären Bewegung selber rekrutiert hatte. Die sroten Direktorens, die sich in ihrem Interesse nach operativer Selbständigkeic auf das rechter und auch auf das theoretisch reflektierte trotzkistische Industrialisierungskonzept der Partei- und Staatsspitze hatten stützen können, wurden bis 1939 durch die nunmehr während des zweiten Fünf-Jahr-Planes auch besser technisch wie ideologisch qualifizierte Sowjecintelligenz ersetzt. Als der XVIII. Parteitag im Jahre 1939 stattfand, war die Herrschaftssynthese beider Intelligenzfraktionen weitgehend abgeschlossen. Die neue Intelligenz zog in die höchsten Parteiämter ein. Beide Fraktionen wurden nun von der Partei als Hegemonialorganisation der geistig-synchetischen Arbeit verklammer. ${ }^{1 \infty}$ Der sozialstrukturelle Austausch von Herrschaftsfrakrionen entsprach den organisatorischen Anforderungen des neuen staatlich-bürokratischen Vergesellschafungs-

96 Ebenda; in gleicher Weise argumenciere R. Lorenz, Poliuscher Terror in der UdSSR, in: Das Argument 21. Jg., Nr. 114 .

97 P. Schulze, a. a. O., S. r 1. Schulze häle gleiehwohl wenig überzeugend an dem schwammigen Begriff der - Ubergangsgesellschafi a lest.

98 Vgl. P. Schulze, a. a. O., S. $99 \mathrm{ft}$.

99 Die empirischen Grundlagen dieser These Schules können in diesem Rahmen nicht überprüft wcrden. Sie ariod hier als Erkelärungshypochese eingeführt Sic wäre durch weitere Forschungen zu untermauern. 10 So P. Schulze, a. a. O., S. 143 . 
typus. Die Herstellung der gesellschaftlichen Synthesis nach Maßjgabe verwaltungsrechtlicher Regelungen erforderte nicht nur eine personelle Ausdehnung des Staatsapparates, sondern auch eine personelle Homogenisienung. Mit der Gesampplanung der Okonomie übernahm der Staat zugleich die Generalverantwortung für alle sozial norwendigen Produkcions- und Reproduktionsfunktionen. Nur die bedingungslose Loyalität seiner funktionalen Einzelgliederungen konnte die gewaltigen Legitimitätsanforderungen, die er damit auf sich nahm, erträglich machen. Jede personelle Fehlfunkrion konnce nunmehr regionale oder gesamtindustrielle Störungen hervorrufen. Wo solche Störungen nicht ausblieben, konnte nur die ganze Repressivitäc des Strafrechts die notwendig eintretenden Legitimationsverluste gleichsam kompensieren. Die Geschichte der politischen Justiz ist damit zugleich die Geschichte einer neuen sowjerischen Herrschaftsstrukrur.

Der CILIP.Inlormationsdiensi erscheint jewells in einer eng. lischen und einer deutschen Ausgabe dreimal pro Jahr

Preis pro Einzelhelt: DM 6.- plus Versandkosten

Abonnement: Personen DM 20.- incl. Versandkosten Institutionen: DM 30.- incl. Versandkosten

Redaktionsadresse: CILIP, clo Berghofstiltung for Konllikt. forschung, Winklerstr. 4a, 1000 Berlin 33 